\title{
DISENTANGLING THE EFFECTS OF OBJECT POSITION AND MOTION ON HEADING JUDGMENTS IN THE PRESENCE OF A MOVING OBJECT
}

Long Ni (nilgucas@hotmail.com),Diederick Niehorster, Li Li

\author{
Department of Psychology, The University of Hong Kong, Pokfulam, Hong Kong SAR, China
}

Previous research has found that moving objects bias heading perception only when they occlude the focus of expansion (FOE) in the background optic flow, with the direction of bias depending on whether the moving object was approached or at a fixed distance from the moving observer. However, the effect of object motion on heading perception was confounded with object position in previous studies. Here, we disentangled the contributions of object motion and position to heading bias. In each $1 \mathrm{~s}$ trial, the display simulated forward observer motion at $1 \mathrm{~m} / \mathrm{s}$ through a $3 \mathrm{D}$ random-dot cloud that had a constant depth range of $0.5-2 \mathrm{~m}$ and consisted of 50 dots which were always seen in the field of view. In Experiment 1, an opaque square that had a fixed position on the screen contained 9 dots that moved uniformly laterally or in random directions at a fixed distance from the observer. In Experiment 2, the dots in the square had the same lateral motion component as in Experiment 1, but simultaneously approached the moving observer at $1 \mathrm{~m} / \mathrm{s}$. The distance between the center of the square and the FOE $\left( \pm 5^{\circ}\right.$ or $\left.\pm 10^{\circ}\right)$ was constant throughout the trial. We found that heading perception was biased even when the FOE was not occluded and the bias was in the direction of object motion when the object was at a fixed distance from the moving observer and was in the opposite direction when it was approached in depth. In addition, when the object contained random motion (Experiment 1), heading bias was toward the object position. We conclude that occlusion of the FOE is not a prerequisite for moving objects to induce a heading bias, consistent with Royden's (2002) differential motion model, and that the bias can be due to either object motion or position.

Acknowledgement: Hong Kong Research Grant Council, HKU 7460/13H, 7482/12H. 